\title{
Polymorphic microsatellites for the lesser bulldog bat (Noctilio albiventris) cross-amplify with close and distant relatives
}

\author{
Dina K. N. Dechmann - Lena John . \\ Tanja Noventa $\cdot$ Christian C. Voigt
}

\begin{abstract}
We used the enriched genomic library to isolate 16 polymorphic microsatellite markers from the lesser bulldog bat (Noctilio albiventris). We analysed DNA from 226 individuals from two neighbouring populations to assess genetic polymorphism at these loci. Polymorphism varied from two to ten alleles per locus, with expected heterozygosity ranging from 0.309 to 0.766 (with the exception of one for which no $P$-value was calculated). Two loci, including the one mentioned above, departed from Hardy-Weinberg equilibrium and linkage disequilibrium was detected between one locus with two other loci after Bonferroni correction. All loci cross-amplified successfully with a member of at least one other bat family, several of them polymorphically. These loci will be used to study relatedness and parentage in a study on the social system of Noctilio albiventris.
\end{abstract}

Keywords Microsatellite loci - Population structure * Relatedness - Social system

The lesser bulldog bat, Noctilio albiventris, commonly occurs from Northern Honduras all the way down to Paraguay and the East coast of Brazil (Hood and Pitocchelli 1983). It naturally roosts in tree cavities, but also commonly inhabits buildings (Reid 1997). It forages in groups

D. K. N. Dechmann - L. John - T. Noventa - C. C. Voigt Leibniz Institute for Zoo and Wildlife Research, Alfred-Kowalke-Str. 17, 10315 Berlin, Germany

\section{Present Address:}

D. K. N. Dechmann (ब)

Department of Omithology, University of Konstanz,

78457 Constance, Germany

e-mail: ddechmann@orn.mpg.de for swarming insects over open water bodies (Dechmann et al. published online; Hood and Pitocchelli 1983) As a basis for a project studying the reasons for sociality in bats, we developed and tested microsatellite primers for this species (primer development by Genetic Identification Services, Chatsworth, California USA).

Methods for DNA library construction, enrichment and screening were as described previously (Jones et al. 2002). Genomic DNA was partially restricted with a cocktail of seven blunt-end cutting enzymes (Rsa I, Hae III, Bsr B1, Pvu II, Stu I, Sca I, Eco RV). Fragments in the size range of $300-750$ bp were adapted and subjected to magnetic bead capture (CPG, Inc., Lincoln Park, New Jersey), using biotinylated capture molecules.

Libraries were prepared in parallel using BiotinAAC(12), Biotin-ATG(12), Biotin-CATC(8) and BiotinTAGA(8) as capture molecules in a protocol provided by the manufacturer. Captured molecules were amplified and restricted with HindIII to remove the adapters. The resulting fragments were ligated into the HindIII site of pUC19. Recombinant molecules were electroporated into E. coli DH5alpha. Recombinant clones were selected at random for sequencing, and enrichment levels were expressed as the fraction of sequences that contained a microsatellite. Sequences were obtained on an ABI 377, using ABI Prism Taq dye terminator cycle sequencing methodology.

The optimal amplification reaction mix for all primer pairs consisted of $1 \times$ BiolaseC Buffer (from $10 \times$ stock solution supplied by manufacturer), $2 \mathrm{mM} \mathrm{MgCl}, 0.2 \mathrm{mM}$ each dNTP's, $6 \mathrm{M}$ each primer (forward primer fluorescent-labeled), $0.025 \mathrm{U} / \mathrm{l}$ BiolaseC Taq polymerase, and $0.2 \mathrm{ng} / \mathrm{t}$ template DNA in $50 \mu \mathrm{l}$ final reaction volume. Samples were amplified in a Perkin-Elmer-Cetus thermal cycler by an initial three minutes of denaturation at $94^{\circ} \mathrm{C}$, 
Table 1 Primer pairs for 16 microsatellite loci derived from Noctilio albiventris. Locus size and repeat motif are derived from the original clone sequenced. Number of alleles are those detected in 226 samples from Barro Colorado Island and Gamboa, Panama. $H_{\mathrm{E}}$ Expected heterozygosity, $H_{\mathrm{O}}$ Observed heterozygosity

\begin{tabular}{|c|c|c|c|c|c|c|c|c|c|}
\hline Locus & Primersequence $\left(5^{\prime}-3^{\prime}\right)$ & $\begin{array}{l}\text { Annealing } \\
\text { temp. }\left({ }^{\circ} \mathrm{C}\right)\end{array}$ & Repeat motif & Locus size (bp) & $\begin{array}{l}\text { Number of } \\
\text { alleles detected }\end{array}$ & $H_{0}$ & $H_{\mathrm{E}}$ & $P$-value & $\begin{array}{l}\text { GenBank } \\
\text { Accession No. }\end{array}$ \\
\hline NA A 103 & $\begin{array}{l}\text { GGC-ACT-ATC-AGA-GAT-GTC-CC } \\
\text { GGA-AGG-AAG-CCA-GGA-TTC }\end{array}$ & 56.7 & $(\mathrm{AAC})_{7}$ & 177 & 3 & 0.450 & 0.432 & NS & GQ183812 \\
\hline NA A 108 & $\begin{array}{l}\text { CAG-GGA-ACA-TAG-CAG-CTC-TAC } \\
\text { CTT-TCT-TTC-ATT-TGG-GAT-CTG }\end{array}$ & 55.3 & $(\mathrm{AAC})_{7}$ & 122 & 4 & 0.296 & 0.309 & NS & GQ183813 \\
\hline NA B 1 & $\begin{array}{l}\text { TGT-GGA-ATG-CCC-AGT-AAT-G } \\
\text { GTC-ACG-GTT-GCC-AGA-AAG }\end{array}$ & 58 & $(\mathrm{CAT})_{12}$ & 292 & 4 & 0.542 & 0.543 & NS & GQ183814 \\
\hline NA B4 & $\begin{array}{l}\text { GCC-CAC-TGT-GCT-ATC-TTT-C } \\
\text { ACC-AGG-CTA-TTC-ACT-CAG-C }\end{array}$ & 56.8 & $(\mathrm{TGA})_{13}$ & 220 & 5 & 0.695 & 0.703 & NS & GQ183815 \\
\hline NA B 108 & $\begin{array}{l}\text { ATC-CCT-TTC-GCT-CTG-ATI-AG } \\
\text { TGG-CTC-ATA-AGT-GGT-CAC-AT }\end{array}$ & 57.4 & $(\mathrm{CAT})_{9}$ & 269 & 4 & 0.609 & 0.662 & NS & GQ183816 \\
\hline $\mathrm{NAC} 2$ & $\begin{array}{l}\text { AAA-ACC-TTT-CCA-CCT-TTG-AGT } \\
\text { ATG-CGA-AGT-AAA-TGT-GTG-ATG }\end{array}$ & 57.5 & $(\mathrm{ATTC})_{1,}$ & 281 & 4 & 0.486 & 0.565 & NS & GQ183817 \\
\hline NA C 8 & $\begin{array}{l}\text { GGT-GAG-GGC-AGG-TTA-GTC } \\
\text { TGA-GCA-GAT-AGA-GAT-GGA-CAG }\end{array}$ & 56.8 & $(\mathrm{CATC})_{7}$ & 224 & 4 & 0.511 & 0.497 & NS & GQ183818 \\
\hline $\mathrm{NACl}$ & $\begin{array}{l}\text { ATC-CAC-CCA-TCC-ATC-TGT-TC } \\
\text { GCC-CAA-CCT-ACT-TGC-TCA-AC }\end{array}$ & 56.6 & $(\mathrm{TCCA})_{11}$ & 133 & 4 & 0.463 & 0.473 & NS & GQ183819 \\
\hline $\mathrm{NACl}$ & $\begin{array}{l}\text { TTA-GGC-ATC-ACT-CTC-ACT-TGG } \\
\text { CTC-CAG-CCA-CAG-GAC-TTT-AC }\end{array}$ & 57.3 & $(\mathrm{CATC})_{6}$ & 208 & 3 & 0.441 & 0.479 & NS & GQ183820 \\
\hline $\mathrm{NACl}$ & $\begin{array}{l}\text { ACG-TGG-AAT-AAC-TGG-TTC-CC } \\
\text { GAT-GGA-TGG-ATG-GAT-GAG-TG }\end{array}$ & 58.1 & $(\mathrm{CATC})_{7}$ & 268 & 2 & 0.013 & 0.013 & - & GQ183821 \\
\hline NA D8 & $\begin{array}{l}\text { GGT-GAT-TCC-TGA-ATT-GAC-TG } \\
\text { CCC-TTA-GGT-AAG-AAA-ACT-ACC-C }\end{array}$ & 56.3 & $(\mathrm{GATA})_{2} \mathrm{CATA}(\mathrm{GATA})_{6}$ & 183 & 2 & 0.502 & 0.498 & NS & GQ183822 \\
\hline NA D9 & $\begin{array}{l}\text { GCA-TAA-AAT-GAG-GCA-GTC-AG } \\
\text { TCA-CCC-AGG-AAA-TAC-AAG-G }\end{array}$ & 57.0 & $(\mathrm{TCTA})_{7} \mathrm{TC}(\mathrm{TCTA})_{13}$ & 225 & 6 & 0.811 & 0.736 & NS & GQ183823 \\
\hline NA D 103 & $\begin{array}{l}\text { GAA-AAG-AGC-GGA-GGA-AGA-G } \\
\text { GGA-GAA-CAG-ACT-AAA-GGT-GGA-C }\end{array}$ & 56.3 & $(\mathrm{TATC})_{11}$ & 157 & 6 & 0.763 & 0.739 & NS & GQ183824 \\
\hline NA D107 & $\begin{array}{l}\text { CGC-TTG-GGA-GAT-TAC-ATA-CAT-C } \\
\text { TGC-AGC-AGT-AAC-CAC-ATT-TAT-G }\end{array}$ & 58.2 & $(\mathrm{GATA})_{12}$ & 283 & 10 & 0.647 & 0.763 & ** & GQ183825 \\
\hline NA D116 & $\begin{array}{l}\text { CCT-CAA-GGC-TAG-GTC-TCT-GAG } \\
\text { CCA-AGG-GTC-CTT-ATG-ACT-TTG }\end{array}$ & 56.5 & $(\mathrm{GATA})_{12}$ & 146 & 6 & 0.765 & 0.766 & NS & GQ183826 \\
\hline NA D 118 & $\begin{array}{l}\text { TGT-GGT-GTA-GAA-ATG-CTA-CTT-G } \\
\text { ATG-GGC-ATC-TGA-AGG-TAT-TT }\end{array}$ & 56.9 & $(\mathrm{ATCT})_{11}$ & 234 & 4 & 0.590 & 0.591 & NS & GQ183827 \\
\hline
\end{tabular}


Table 2 Cross-species amplification tests with eight species from five families, using the primers developed for Noctilio albiventris minor from Panama

\begin{tabular}{|c|c|c|c|c|c|c|c|c|c|c|c|c|c|c|c|c|c|}
\hline Species $(n)$ & Family & $\begin{array}{l}\text { NA } \\
\text { A103 }\end{array}$ & $\begin{array}{l}\text { NA } \\
\text { A108 }\end{array}$ & $\begin{array}{l}\text { NA } \\
\text { B1 }\end{array}$ & $\begin{array}{l}\text { NA } \\
\text { B4 }\end{array}$ & $\begin{array}{l}\text { NA } \\
\text { B } 108\end{array}$ & $\begin{array}{l}\mathrm{NA} \\
\mathrm{C} 2\end{array}$ & $\begin{array}{l}\mathrm{NA} \\
\mathrm{C} 8\end{array}$ & $\begin{array}{l}\text { NA } \\
\text { C101 }\end{array}$ & $\begin{array}{l}\text { NA } \\
\text { C104 }\end{array}$ & $\begin{array}{l}\text { NA } \\
\text { C114 }\end{array}$ & $\begin{array}{l}\text { NA } \\
\text { D8 }\end{array}$ & $\begin{array}{l}\text { NA } \\
\text { D9 }\end{array}$ & $\begin{array}{l}\text { NA } \\
\text { D103 }\end{array}$ & $\begin{array}{l}\text { NA } \\
\text { D107 }\end{array}$ & $\begin{array}{l}\text { NA } \\
\text { D116 }\end{array}$ & $\begin{array}{l}\text { NA } \\
\text { D118 }\end{array}$ \\
\hline $\begin{array}{l}\text { Saccopteryx } \\
\text { bilineata (3) }\end{array}$ & Emballonuridae & 1 & 2 & 1 & 2 & 0 & 0 & 0 & 3 & 2 & 0 & 1 & 1 & 3 & 0 & 0 & 2 \\
\hline Molossus molossus ( 3 ) & Molossidae & 1 & 1 & 2 & 2 & 1 & 0 & 2 & 4 & 2 & 2 & 2 & 1 & 2 & 0 & 0 & 0 \\
\hline Pteronotus parnellii (5) & Mormoopidae & 1 & 2 & 1 & 2 & 0 & 1 & 3 & 2 & 2 & 1 & 1 & 2 & 2 & 0 & 2 & 2 \\
\hline $\begin{array}{l}\text { Noctilio albiventris } \\
\text { affinis (1) }\end{array}$ & Noctilionidae & 1 & 0 & 2 & 2 & 1 & 0 & 1 & 0 & 1 & 0 & 2 & 2 & 0 & 1 & 2 & 1 \\
\hline Noctilio leporinus (6) & Noctilionidae & 2 & 1 & 2 & 2 & 2 & 2 & 1 & 3 & 1 & 1 & 2 & 5 & 6 & 1 & 1 & 4 \\
\hline $\begin{array}{l}\text { Carollia } \\
\quad \text { perspicillata (8) }\end{array}$ & Phyllostomidae & 1 & 2 & 1 & 1 & 1 & 1 & 1 & 4 & 2 & 4 & 3 & 2 & 5 & 1 & 0 & 0 \\
\hline Vampyressa pusilla (1) & Phyllostomidae & 2 & 1 & 0 & 1 & 0 & 0 & 0 & 1 & 2 & 1 & 1 & 1 & 2 & 0 & 0 & 0 \\
\hline
\end{tabular}

Number of alleles given $(0$, unsuccessful cross-species amplification; 1 , successful cross-species amplification, but no polymorphism found among the analysed individuals; $2-6$. polymorphic, number of alleles given)

followed by 35 cycles of denaturation $\left(94^{\circ} \mathrm{C}, 40 \mathrm{~s}\right)$, annealing $\left(55^{\circ} \mathrm{C}, 40\right.$ seconds), and extension $\left(72^{\circ} \mathrm{C}, 30 \mathrm{~s}\right)$, with final extension time of $4 \mathrm{~min}$ at $72^{\circ} \mathrm{C}$.

DNA was extracted using the PureGene DNA Extraction KitR kit (Gentra Systems, Minneapolis, MN, USA) following the manufacturer's instructions. Microsatellite loci were amplified in $10 \mu \mathrm{l}$ reactions in the following reaction mix: $\mathrm{MgCl}_{2}, 2 \mathrm{mM}$; dNTPs (premixed), $0.2 \mathrm{mM}$ each; primers, $0.3 \mu \mathrm{M}$ each; BioTaq DNA PolymeraseR (Bioline USA, Canton, MA, USA), $0.025 \mathrm{U} / \mu \mathrm{l}$; template DNA, $0.2 \mathrm{ng} / \mu \mathrm{l}$. PCR was conducted in a RoboCycler Gradient 96R thermocycler (Stratagene, Inc., La Jolla, CA, USA) by an initial denaturation $\left(94^{\circ} \mathrm{C}, 3 \mathrm{~min}\right)$, followed by 35 cycles of denaturation $\left(94^{\circ} \mathrm{C}, 40 \mathrm{~s}\right)$, annealing $\left(55^{\circ} \mathrm{C}, 40 \mathrm{~s}\right)$, and extension $\left(72^{\circ} \mathrm{C}, 30 \mathrm{~s}\right)$, and a final extension at $72^{\circ} \mathrm{C}$ for $4 \mathrm{~min}$. PCR products were labeled using one of the conventional sequencing dyes NED, HEX or FAM (Applied Biosystems. Inc.). Amplification products were separated on polyacrylamide gels in an ABI 377 DNA sequencer and sized using Genotyper 2.5 software and Rox $400 \mathrm{HD}$ size markers (Applied Biosystems, Inc., Foster City, CA USA).

To test our microsatellite primers for applicability, 226 individual lesser bulldog bats, Noctilio albiventris, were captured in the village Gamboa (N $09.07^{\circ}$; W $079.41^{\circ}$ ) in Panama, and at surrounding water bodies. An additional capture site was Barro Colorado Island (BCI, N 09.10 $079.51^{\circ}$ ). Tissue samples were taken from the wing membrane with a sterile, $3 \mathrm{~mm}$ diameter biopsy punch (Stiefel; Germany; Worthington-Wilmer and Baratt 1996) and stored in $99 \%$ alcohol until extraction. Extracted samples from additional species (Saccopteryx bilineata (family Emballonuridae), Molossus molossus (Molossidae) Pteronotus parnellii (Mormoopidae), Noctilio leporinus
(Noctilionidae) Carollia perspicillata, and Vampyressa pusilla (both Phyllostomidae), were already available from previous projects, and were used for crossamplification. Finally, one sample of the subspecies Noctilio albiventris affinis from Tiputini Biological Station Ecuador was also included in the analysis. DNA was extracted in the laboratory with the help of the QIAamp DNA Mini Kit ${ }^{\mathrm{TM}}$ and stored in purified water.

All amplifications were performed using one fluorescently labeled forward primer for each locus (6-Fam, locus NA A108, NA B1, NA C8, NA C104, NA D8, NA D107, NA D118; Hex, NA A103, NA B4, NA B108, NA C2, NA C101, C114, NA D9, NA D103, NA D116; Biotez, Berlin, Germany). PCRs were carried out in $10 \mu \mathrm{l}$ reaction volumes containing $1 \mu \mathrm{l}$ of undiluted DNA, $5 \mu \mathrm{l}$ of Qiagen Multiplex mix (Qiagen Europe, Hilden, Germany), and $0.25 \mu \mathrm{l}$ of each primer filling up the volume to $10 \mu \mathrm{l}$ with RNase free water (Qiagen). Reactions were cycled using a Primus Gradient (MWG AG Biotech) or a 2720 Thermal Cycler (Applied Biosystems) and the following thermotreatment: $15 \mathrm{~min}$ at $94^{\circ} \mathrm{C}$, followed by 30 cycles of $30 \mathrm{~s}$ at $94^{\circ} \mathrm{C}, 90 \mathrm{~s}$ at $58^{\circ} \mathrm{C}$ (adjusting the temperatures was not necessary when using the Multiplex mix), and $90 \mathrm{~s}$ at $72^{\circ} \mathrm{C}$. All thermotreatments were concluded with a $30 \mathrm{~min}$ extension at $60^{\circ} \mathrm{C}$. After amplification, $0.8 \mu \mathrm{l}$ of $1: 4$ diluted PCR product were electrophoresed in $10 \mu \mathrm{l}$ formamide (Hi-Di ${ }^{\text {TM }}$ Formamide, Applied Biosystems) and $0.5 \mu \mathrm{l}$ rox standard (GeneScan ${ }^{\mathrm{TM}}-500 \mathrm{ROX}^{\mathrm{TM}}$ Size Standard, Applied Biosystems) using a $\mathrm{ABI} 3130 \mathrm{xl}$ Genetic Analyzer (Applied Biosystems) with a $36 \mathrm{~cm}$ 16-capillary Array $(47 \mathrm{~cm} \times 50 \mu \mathrm{m})$. Running conditions were as follows: Injection time, $5 \mathrm{~s}$, injection voltage $1.2 \mathrm{KV}$, run time $880 \mathrm{~min}$, run voltage $15 \mathrm{KV}$. Fragment analysis was completed using GeneMapper v. 3.7 (Applied Biosystems). 
Expected and observed counts for heterozygotes, deviations from Hardy-Weinberg equilibrium, and linkage disequilibrium were calculated using Cervus 3.0.3 (Marshall et al. 1998) and Genepop 4.0, option 2 (Raymond and Rousset 1995).

We found between two and ten alleles per primer among our animal sample. All but NA D107 were in HardyWeinberg equilibrium (Table 1). For another primer, NA $\mathrm{C} 114$, which has only two alleles $\mathrm{H}_{\mathrm{c}}$ was far below 0.5 and Hardy-Weinberg was not calculated. However as this primer was polymorphic in two other species ( $C$. perspicillata, 4 alleles: $M$. molossus, 2 alleles; Table 2), it is nonetheless listed in the tables here, but was not used for analysis with $N$. albiventris. In fact, all of the microsatellites developed for $N$. albiventris cross-amplified with other species, some of them polymorphically (Table 2). We detected no linkage disequilibrium after Bonferroni except between NA C 2 and NA C104 and between NA C 2 and NA D116. Thus, the primer sets presented here do not offer a basis for the analysis of parentage and population structure in $N$. albiventris, but also potentially for the genetic study of other species and even families of bats.

Acknowledgements We thank Arne Ludwig for help with the interpretation of results. This study was financed by the German
Science Foundation, grant number (Vo 890/11) to CC Voigt and DKN Dechmann.

\section{References}

Dechmann DKN, Heucke SL, Giuggioli L. Safi K, Voigt CC, Wikelski M (2009) Experimental evidence for group hunting via eavesdropping in echolocating bats. Proc R Soc B. doi:10.1098/ rspb.2009.0473

Hood CS, Pitocchelli J (1983) Noctilio albiventris. Mamm Species 197:1-5

Jones KC, Levine KF, Banks JD (2002) Characterization of 11 polymorphic tetranucleotide microsatellites for forensic applications in California elk (Cervus elaphus canadensis). Mol Ecol Notes 2:425-427

Marshall TC, Slate J, Kruuk LEB, Pemberton JM (1998) Statistical confidence for likelihood-based paternity inference in natural populations. Mol Ecol 7:639-655

Raymond M, Rousset F (1995) GENEPOP (version 1.2): population genetics software for exact tests and ecumenicism. J Hered 86 : 248-249

Reid FA (1997) A field guide to the mammals of Central America and Southeast Mexico. Oxford University Press, New York, Oxford

Worthington-Wilmer J, Baratt E (1996) A non-lethal method of tissue-sampling for genetic studies of chiropterans. Bat Research News $37: 1-3$ 